\title{
A Comparison of Phase-Preserving Algorithms for Burst-Mode SAR Data Processing
}

\author{
Ian Cumming and Yi Guo* \\ Dept. of Electrical and Computer Engineering \\ The University of British Columbia, Vancouver, BC, Canada V6T 1 Z4. \\ tel: 604-822-4623 fax: 604-822-5949 ianc@ee.ubc.ca yig@ee.ubc.ca

\section{Frank Wong} \\ MacDonald Dettwiler and Associates, Richmond, B.C., Canada \\ tel: 604-278-3411 fax: 604-278-0531 fhw@mda.ca
}

\begin{abstract}
Burst-mode data is obtained when a SAR system is operated in ScanSAR mode. Missing data in the burst gaps makes it difficult to use traditional phase-preserving algorithms to produce single look complex (SLC) data. In this paper, three phase-preserving algorithms for burst-mode data compression are examined and compared to find the best algorithm for precision processing applications such as interferometry.
\end{abstract}

\section{INTRODUCTION}

With the increasing interest in SAR interferometry (InSAR), it is important that SAR processing algorithms be phase preserving. In the last few years, many sophisticated phase-preserving SAR algorithms, such as classical Range Doppler (RD), modified RD, SPECAN (de-ramp and FFT) and chirp scaling have been published. These algorithms work well on continuous-mode data, but have to be modified when applied to burst-mode data, because the spectrum of the data varies with time.

This paper will discuss three phase-preserving algorithms for burst-mode data, two modified from the RD algorithm, and one from the SPECAN algorithm. We show the simulation results using these algorithms, and by comparing these results, the best algorithm will be recommended.

\section{BURST-MODE DATA}

Burst-mode data is obtained when SAR works in a ScanSAR mode. In this mode, the satellite antenna scans through different range subswaths in order to image a larger area. In cach subswath, the received range signal is the same as the continuous range signal, however, in azimuth direction, the data is blocked into bursts.

\footnotetext{
*This work is supported by the NSERC/MDA chair in Radar Remote Sensing.
}

Fig. 1 shows the difference between continuous-mode and burst-mode data. The Doppler spectrum of a continuous-mode target covers the full antenna bandwidth, the same for each target. However, in burst mode, different targets will have a different spectral distribution, depending on the target's azimuth location.

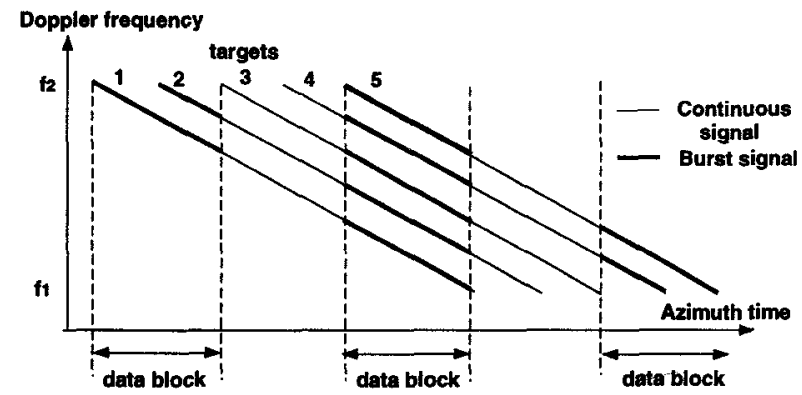

Figure 1: Target spectral distribution in continuous and burst mode

This discontinuous, non-stationary Doppler spectrum makes it difficult to apply traditional phase preserving compression algorithms. Modifications of the algorithms are necessary for the compression of burst-mode data.

\section{MODIFIED RD ALGORITHMS}

In this section, two modifications of the RD algorithm will be discussed.

\subsection{The range/Doppler algorithm}

The traditional range/Doppler (RD) algorithm consists of the following steps [1, 2]:

1. Range compression.

2. Azimuth FFT.

3. Range cell migration correction (RCMC). 
4. Azimuth matched filter multiplication.

5. Complete azimuth compression using IFFTs.

In continuous mode, the compressed signal from the RD algorithm has stationary phase properties: the phase slope is constant for all the targets, and at the peak, the phase information of the target is preserved. When data is collected in burst mode, as the effective Doppler centroid frequency varies with targets, the RD algorithm will result in a non-stationary compressed signal.

\subsection{Bamler's algorithm}

Bamler's algorithm directly applies the RD algorithm to the burst-mode data [3]. If a point-target azimuth signal is: 1

$$
s(\eta)=\sum_{i} \operatorname{rect}\left(\frac{\eta-\eta_{b c}(i)}{T_{b}}\right) \exp \left(-j \pi K_{a}\left(\eta-\eta_{\mathfrak{a}}\right)^{2}\right)
$$

where $K_{a}$ is the azimuth FM rate, $\eta_{a}$ is the zero Doppler time of the target, $\eta_{b c}$ is the time of the centre of the burst, and $T_{b}$ is the burst duration, Bamler's compression result is the same as coherently adding the RD compression results of all the bursts.

The compressed pulse of a single burst is a sinc function, its phase has a linear slope depending on the burst center frequency and a negligible quadratic component, and phase is preserved at the peak (the peak phase is zero). When such pulses from all bursts are added coherently, the resulting pulse still preserves the phase at the peak, but the magnitude curve is spiky due to the different phase slopes from each burst. This compressed phase slope at the peak is proportional to the target's effective Doppler centroid frequency, however the phase value frequently jumps by $\pm \pi$ whenever the amplitude crosses zero.

\subsection{The SIFFT algorithm}

The SIFFT algorithm developed at MDA [4] is also a modification of the $\mathrm{RD}$ algorithm. At the last step of the $\mathrm{RD}$ algorithm, part of the spectrum is extracted using a short IFFT, and by doing several such short IFFTs, the complete array of compressed targets can be obtained by stitching the short IFFT results together.

If $f_{1}$ and $f_{2}$ are the burst spectrum boundaries, and the short IFFT is taken between $f_{a}$ and $f_{b}$, where $f_{a} \leq$ $f_{1}<f_{2} \leq f_{b}$, then the SIFFT output from this burst after phase compensation is:

$$
\begin{aligned}
c_{1}(\eta)= & \exp \left[j \pi\left(K_{a}\left(\eta-\eta_{a}\right)^{2}-\left(\eta-\eta_{a}\right)\left(f_{2}+f_{1}\right)\right)\right] \\
& \operatorname{sinc}\left[\left(\eta-\eta_{a}\right)\left(f_{2}-f_{1}\right)\right]
\end{aligned}
$$

\footnotetext{
${ }^{1}$ A phase term due to the slant range $R_{0}$ and its amplitude which is only a multiplicative constant are ignored in equations (1) to (3).
}

This compressed pulse is a sinc function with resolution depending on the burst bandwidth, $\left(f_{2}-f_{1}\right)$. It preserves the phase at the compressed peak. Also, the phase is a linear ramp with a slope proportional to the burst center location, plus a small quadratic component.

\section{SPECAN ALGORITHM}

The generic SPECAN algorithm includes deramping and DFT operations [5, 6]. When applied to burst-mode data, the reference function is the same, and the DFT is simply aligned to each burst segment.

Using the same example as in the SIFFT algorithm, in which $f_{1}=K_{a}\left(\eta_{1}-\eta_{a}\right)$, and $f_{2}=K_{a}\left(\eta_{2}-\eta_{a}\right)$, where $\eta_{1}$ and $\eta_{2}$ are the burst boundaries in azimuth time, and the de-ramp function orgin is at $\eta=0$, the SPECAN output after phase compensation is:

$$
\begin{aligned}
c_{2}(\eta)= & \exp \left[j \pi K_{a}\left(\left(\eta-\eta_{a}\right)^{2}-\left(\eta-\eta_{a}\right)\left(\eta_{1}+\eta_{2}-2 \eta_{a}\right)\right)\right] \\
& \operatorname{sinc}\left[K_{a}\left(\eta-\eta_{a}\right)\left(\eta_{2}-\eta_{1}\right)\right]
\end{aligned}
$$

As with the SIFFT algorithm, the phase at the peak of the compressed pulse is preserved, and a phase slope proportional to the target-dependent burst Doppler centroid is present at the peak. In addition, similar to the SIFFT algorithm, a small quadratic phase term exists.

\section{SIMULATION RESULTS}

The above three algorithms are all modified from algorithms for continuous data. By compressing only one burst for each target, both the SIFFT and SPECAN results results show a linear phase plus a small quadratic component. On the other hand, the Bamler's algorithm compresses the whole spectrum of a target at once, but gives a spiky result. In this section, we will compare the simulation results of the three algorithms.

\subsection{The SIFFT and SPECAN algorithms}

Here the simulation results of the SIFFT algorithm and the SPECAN algorithm are compared. Consider a target exposed by 3 bursts, with a distribution of spectral energy shown in Fig. 2. We compute the compressed pulse properties from IFFT2 in the SIFFT method, and from the second burst in the SPECAN method.

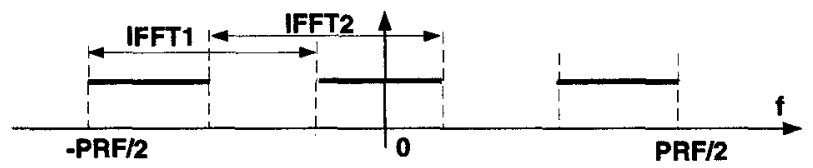

Figure 2: Doppler spectrum of a burst-mode target

The magnitude curves of the two results are the same, as the resolution is related to the burst bandwidth in each 

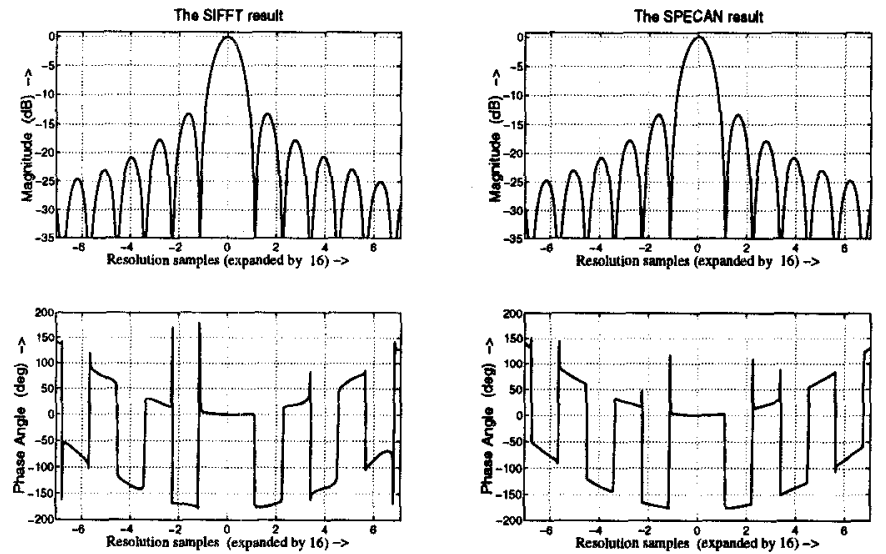

Figure 3: The compressed pulses from the SIFFT algorithm (left) and the SPECAN algorithm (right)

case. The phase is preserved at the peak of the pulse in each case. Since in this example, the center frequency of the compressed burst spectrum is at the origin, the phase slope at the peak is zero in the two results. As described in the mathematical analysis, the same quadratic component exists in the two results.

It is also interesting to examine the average phase across the peak of the compressed pulse, within the $-3 \mathrm{~dB}$ margins of the peak amplitude. The quadratic phase component takes the average phase away from zero by a small amount in either case. In the current example, the average phase shift is $0.3^{\circ}$.

\subsection{Bamler's and SIFFT algorithms}

Using the same Doppler spectrum in Fig. 2, Bamler's algorithm will compress the whole data at once, with the result shown in Fig. 4. The SIFFT magnitude in Fig. 3 is the envelope of Bamler's result (note that the sampling rate is twice as fast in Fig. 4). The spikes in the compressed pulse makes the phase jump frequently.

The advantage of Bamler's algorithm is that it uses all the information in the data. However in InSAR processing, when bursts are misregistered in the two passes, the interferogram is more susceptible to errors due to a rapidly changing phase, and the spikes will add more noise to the interferogram. The spatial averaging inherent in the SIFF'T algorithm, and the subsequent phase smoothing will give a less noisy result for interferometry use.

\section{CONCLUSIONS}

In this paper, we have analyzed and compared three modified algorithms for precision processing burst-mode data. Bamler's algorithm uses all the data information, but gives a spiky pulse. Comparing these three algorithms, we can conclude that the SIFFT and SPECAN algorithms are favored for interferometric application of
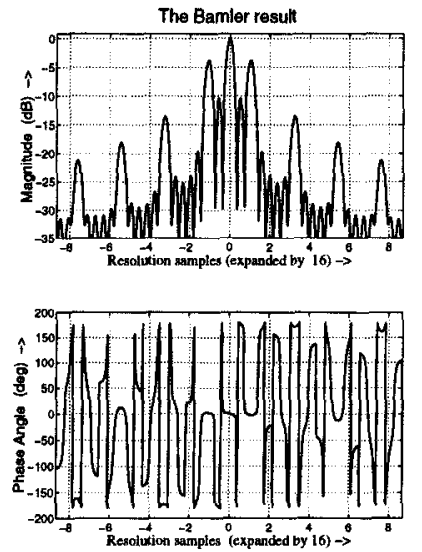

Figure 4: The compressed pulse in Bamler's algorithm

burst-mode data. Out of the two, the SIFFT algorithm has the advantage that it follows the heritage of $\mathrm{RD}$, and that the azimuth spacing in SPECAN is a function of the azimuth FM rate.

\section{References}

[1] I. G. Cumming and J. R. Bennett, "Digital processing of SEASAT SAR data," in IEEE 1979 International Conference on Acoustics, Speech and Signal Processing, (Washington, D.C.), April 2-4, 1979.

[2] C. Wu, K. Y. Liu, and M. J. Jin, "A modeling and correlation algorithm for spaceborne SAR signals," IEEE Trans. on Aerospace and Electronic Systems, vol. AES-18, pp. 563-574, Sept. 1982.

[3] R. Bamler and M. Eineder, "ScanSAR Processing Using Standard High Precision SAR Algorithms," IEEE Trans. Geoscience and Remote Sensing, vol. 34, Jan. 1996.

[4] F. Wong, D. Stevens, and I. Cumming, "PhasePreserving Processing of ScanSAR Data with a Modified Range Doppler Algorithm," in Proceedings of the International Geoscience and Remote Sensing Symposium, IGARSS'97, (Singapore), August 4-8, 1997.

[5] I. Cumming and J. Lim, "The design of a digital breadboard processor for the ESA remote sensing satellite synthetic aperture radar," tech. rep., MacDonald Dettwiler, Richmond, BC, Canada, July 1981. Final report for ESA Contract No. 3998/79/NL/HP(SC).

[6] M. Sack, M. Ito, and I. Cumming, "Application of Efficient Linear FM Matched Filtering Algorithms to SAR Processing," IEEE Proc-F, vol. 132, no. 1, pp. 45-57, 1985. 